\title{
Effect of Two Nonsurgical Periodontal Treatment Modalities in Type 2 Diabetes Mellitus Patients with Chronic Periodontitis: A Randomized Clinical Trial
}

\author{
Tan-Tai Tran, ${ }^{1}$ Quynh-Trang Thi Ngo, ${ }^{2}$ Dang Huu Tran, ${ }^{3}$ Thuy-Duong Thi Nguyen ${ }^{4}$
}

\begin{abstract}
Aim and objective: The current study aimed to investigate the effect of two nonsurgical periodontal treatment modalities on clinical periodontal parameters and glycemic control.

Materials and methods: A randomized clinical trial was conducted with a sample of 64 type 2 diabetes mellitus (T2DM) patients with chronic periodontitis. Subjects were randomly assigned into two groups. Group I received oral hygiene instructions (OHI) at baseline and each recall visit. Group II received one-stage full-mouth scaling and root planning (SRP) combining OHI. At baseline, third month, and sixth month the plaque index (PII), gingival index (GI), probing pocket depth (PPD), clinical attachment loss (CAL), and glycated hemoglobin (HbA1c) were recorded and analyzed.

Results: After treatment, two groups showed significant differences for all parameters at both assessed times $(p<0.05)$. The OHI alone only demonstrated a slight reduction in Gl in third month, with no significant changes for PII and PPD indexes. However, Group I recorded the increased HbA1c and CAL values at 6-month follow-up $(p<0.05)$. The combination of OHI and SRP exhibited a significant improvement in all periodontal values $(p<0.05$ ). Also, the HbA1C levels of Group II showed a significant reduction after treatment and were lower than those of Group I.

Conclusions: Oral hygiene instructions only resulted in a better gingival condition of diabetic patients in the initial time. The nonsurgical periodontal therapy by combining SRP and OHI significantly improved both periodontal health and glycemic control.

Clinical significance: Diabetic patients should be supplied with an effective $\mathrm{OHI}$ modality and a professional dental debridement.

Keywords: Chronic periodontitis, Glycemic control, Nonsurgical periodontal therapy, Type 2 diabetes mellitus.

The Journal of Contemporary Dental Practice (2021): 10.5005/jp-journals-10024-3232
\end{abstract}

\section{INTRODUCTION}

Diabetes mellitus (DM) is one of the prominent health issues of the 21st century, with the number of diabetics tripling over the past 20 years. According to the World Diabetes Association (IDF), 9.3\% of adults aged 20-79 years, equivalent to approximately 463 million adults were living with diabetes. The IDF estimated that this will rise to 578 million people with diabetes by 2030 and 700 million by 2045 . In Vietnam, there were 3.78 million respondents in 2019 and this number is forecasted to increase to 6.34 million by 2045 .

The scientific literature has established the bidirectional relationship between chronic periodontitis $(\mathrm{CP})$ and DM..$^{2,3}$ Diabetes affects periodontal status and periodontitis adversely affects metabolic control, increasing the risk of complications in patients with diabetes. ${ }^{4}$ Some have proposed periodontal disease as the sixth complication of diabetes, over $90 \%$ of diabetic patients have some degree of periodontal tissue damage, both with inflammation and increased oxidative stress as major etiological features. ${ }^{5,6}$ Patients with poor glycemic control have been found to suffer more severe forms of periodontitis. ${ }^{5}$ Many studies have shown that diabetic patients with periodontitis have much worse metabolic control than those with healthy periodontium. ${ }^{7}$

The primary goal of periodontal treatment is to preserve natural dentition by achieving and maintaining a healthy functional periodontium. Nonsurgical therapy is the first in the chronologic sequence of the periodontal treatment procedure. It consists of patient motivation and oral hygiene instructions $(\mathrm{OHI})$ as well as
1,2,4 Faculty of Odonto-stomatology, Hue University of Medicine and Pharmacy, Hue University, Hue, Vietnam

${ }^{3}$ Department of Internal Medicine, Hue University of Medicine and Pharmacy, Hue University, Hue, Vietnam

Corresponding Author: Thuy-Duong Thi Nguyen, Faculty of Odonto-stomatology, Hue University of Medicine and Pharmacy, Hue University, Hue, Vietnam, Phone: +0084 904181688, e-mail: nttduong@hueuni.edu.vn; nttduong@huemed-univ.edu.vn

How to cite this article: Tran TT, Ngo QTT, Tran DH, et al. Effect of Two Nonsurgical Periodontal Treatment Modalities in Type 2 Diabetes Mellitus Patients with Chronic Periodontitis: A Randomized Clinical Trial. J Contemp Dent Pract 2021;22(11):1275-1280.

Source of support: Nil

Conflict of interest: None

mechanical removal of supra- and subgingival plaque, and calculus deposits have a beneficial effect on periodontal tissue healing. ${ }^{8}$ Toward mechanical disruption of the bacterial ecosystem, nonsurgical periodontitis treatment reduces the risk of complications of type 2 diabetes mellitus (T2DM). ${ }^{9}$ The joint workshop between the European and American periodontitis societies in 2013 recommended the need to treat periodontitis in the care regimen of diabetic patients. ${ }^{10}$ The randomized controlled trial results showed that periodontitis treatment improves glycemic control and decreases $\mathrm{HbA1c}$ with good treatment of periodontitis. ${ }^{11}$

oThe Author(s). 2021 Open Access This article is distributed under the terms of the Creative Commons Attribution 4.0 International License (https://creativecommons. org/licenses/by-nc/4.0/), which permits unrestricted use, distribution, and non-commercial reproduction in any medium, provided you give appropriate credit to the original author(s) and the source, provide a link to the Creative Commons license, and indicate if changes were made. The Creative Commons Public Domain Dedication waiver (http://creativecommons.org/publicdomain/zero/1.0/) applies to the data made available in this article, unless otherwise stated. 
Recently, coronavirus disease-19 (COVID-19) is a global pandemic, can lead to severe respiratory infection and death. Santana et al. have reported that uncontrolled hyperglycemia possibly increases the risk of developing periodontitis and triggering overexpression of angiotensin-converting enzyme 2 (ACE2) in periodontal tissue of DM patients. ${ }^{12}$ A study by Morales et al. found that periodontal therapy is likely to improve the oral health-related quality of life in diabetic patients with periodontitis, despite the COVID-19 pandemic. ${ }^{13}$ However, in dental treatment time, the COVID-19 virus can transmit directly and/or indirectly from patients to oral health-care teams through aerosol. ${ }^{14}$ Thus, nonurgent oral health care was advised to be delayed by WHO until there has been a sufficient reduction in virus transmission rates in the community. ${ }^{14}$ Among nonsurgical periodontal therapies, regular scaling and follow-up visits were categorized as elective treatments. ${ }^{15}$ Therefore, many patients have to suspend and postpone the dental treatments, selected to receive remote consultation on maintaining good oral hygiene. ${ }^{14}$

This clinical study was conducted during the COVID-19 pandemic in, some DM patients who selected oral hygiene maintaining at home instead of intensive therapy in clinics. To assess the effect of hygiene instruction on managing periodontal disease associated with DM, two treatment protocols were designed: $\mathrm{OHI}$ alone or in combination with scaling and root planning (SRP). The aim was to evaluate the effect of these two nonsurgical periodontal treatment modalities on glycemic control and periodontal status.

\section{Materials and Methods}

\section{Ethical Approval}

This study was conducted between June 2020 and May 2021 in full accordance with the Helsinki Declaration of 1975, as revised in 2013. The study protocol and the informed consent form were reviewed and approved by the Institutional Ethics Committee of Hue University of Medicine and Pharmacy, Hue University, Hue city, Vietnam (Number: H2020/035). Sixty-four participants were recruited from Hue University of Medicine and Pharmacy Hospital. Consenting participants were presented with a consent form. It was mandatory for consent participants to have read and signed the consent form before being included in the present study.

\section{Study Population}

Participants were recruited from patients who come to the Internal Medicine Clinic, Hue University of Medicine and Pharmacy Hospital for a regular check-up. Subjects diagnosed with T2DM and moderate CP were eligible to participate.

The inclusion criteria were as follows:

- $\geq 40$ years of age;

- Confirmed T2DM for at least 1 year based on criteria given by the American Diabetes Association (ADA, 2020); ${ }^{16}$

- Diagnosis of chronic moderate periodontitis according to the 2014 update Classification of Periodontal Disease by the American Academy of Periodontology; ${ }^{17}$

- Having >15 teeth present per dental arch;

- Ability to perform oral hygiene self-care and commitment to posttreatment follow-up visits.

Exclusion criteria included:

- Pregnancy and lactation,

- Current smoking,

- Periodontal therapy and/or systemic antibiotic therapy in the preceding 6 months,

- Current use of NSAIDs or steroidal anti-inflammatory medications,

- Suspected multiple systemic complications of DM excluding periodontitis, and

- Having acute illness during follow-up.

The selected subjects were randomly allocated to two nonsurgical treatment groups (Flowchart 1):

- Group I (32 patients): subjects who received only OHI.

- Group II (32 patients): subjects who received SRP + OHI.

The patients were scheduled for laboratory and periodontal examinations before treatment (baseline), and after nonsurgical treatment (3-month- and 6-month-recall visits).

Flowchart 1: Schematic diagram of the study design

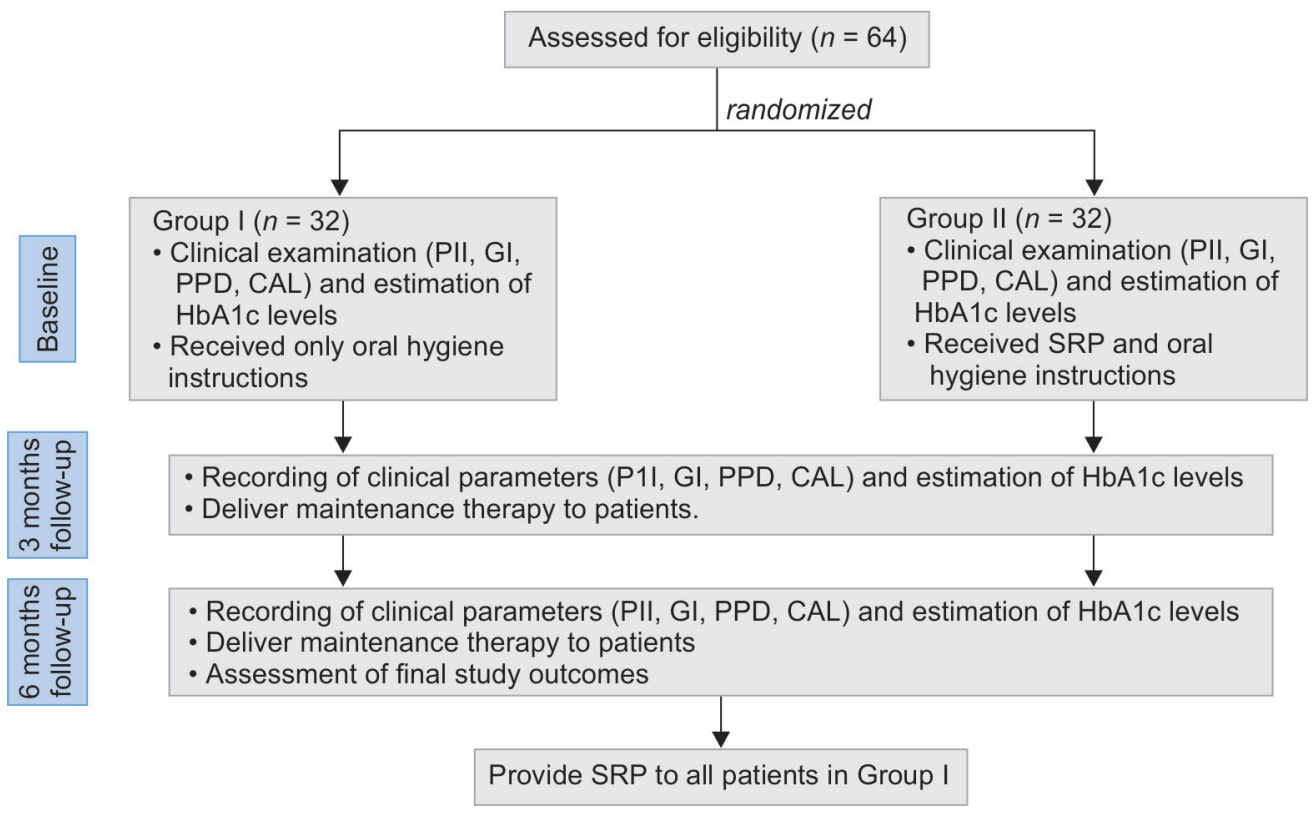




\section{Nonsurgical Treatment}

- Scaling and root planing: Group II patients received a full-mouth scaling and root planing at baseline by performing the supra and subgingival scaling using an ultrasonic scaler and root planning using Gracey curettes.

- Oral hygiene instructions: were given to all patients. Patients were instructed to brush their teeth twice daily using the Bass technique and floss once a day. Oral hygiene control and reinstruction were reviewed each recall visit.

- Other treatments were provided to patients when required:

- Extraction of hopeless teeth.

- Restoration of carious lesions.

- Repair or replacement of poorly fitting restorations and prostheses.

During the trial, diabetes was managed according to clinical guidelines by local specialist diabetes teams, who were unaware of the group assignment throughout the study.

After completing the study, Group I patients were advised and motivated to take SRP treatment.

\section{Method of Obtaining Data}

General information (age, gender, and duration of DM) of each patient were recorded at the first examination.

At baseline, 3 months, and 6 months after treatment, the following clinical parameters were recorded including probing pocket depth (PPD) and clinical attachment loss (CAL) at six sites per tooth. The presence or absence of supragingival dental plaque index (PII) and gingival index (GI) was also recorded. Clinical parameters were carried out on dental chair by a precalibrated examiner who was blinded to the study with the appropriate armamentarium.

Venous blood samples also were taken for each diabetic patient to measure $\mathrm{HbA1c}$ (glycosylated hemoglobin) value at baseline, 3 months, and 6 months after treatment.

\section{Statistical Analysis}

Categorical variable like gender is expressed as proportion and compared by use of Chi-squared test with risk ratios and $95 \%$ confidence intervals quoted.

Quantitative variables were presented as mean \pm standard deviation. The Student $t$-test (two-tailed, independent) was used to find the significance of study parameters on a continuous scale between two groups (intergroup analysis). The student $t$-test (two-tailed, dependent) has been used to find the significance of study parameters on the continuous scale within each group. The Mann-Whitney $U$-test has been used to find the significance of nonparametric parameters probing depth between two groups and the Wilcoxon signed-rank test has been used to find the significance of probing depth (nonparametric) in paired conditions.

All data were collected in an Excel database and analyzed by SPSS version 20.0. A $p$-value of $<0.05$ was considered statistically significant.

\section{Results}

The study sample consisted of 28 men and 36 women (Table 1). The average age of subjects participating in the study was 63.28 \pm 8.86 years. The average duration of DM was $6.6 \pm 4.87$ years. The longest detection time is 20 years, the lowest is 1 year. There was no statistically significant difference between the two groups in terms of age, gender, and time to detect diabetes $(p>0.05)$.
Table 1: Age, gender, duration of diabetes mellitus of the study participants

\begin{tabular}{|c|c|c|c|c|}
\hline Variable & $\begin{array}{l}\text { Group I } \\
\left(n_{1}=32\right)\end{array}$ & $\begin{array}{l}\text { Group II } \\
\left(n_{2}=32\right)\end{array}$ & $\begin{array}{c}\text { Both } \\
(n=42)\end{array}$ & $p$ value \\
\hline $\begin{array}{l}\text { Age } \\
(\text { mean } \pm S D)\end{array}$ & $61.94 \pm 9.00$ & $64.63 \pm 8.64$ & $63.28 \pm 8.86$ & $>0.05^{*}$ \\
\hline Male:female & $14: 18$ & $14: 18$ & $28: 36$ & $>0.05^{*}$ \\
\hline $\begin{array}{l}\text { Duration of } \\
\text { DM (years) } \\
\text { (mean } \pm \text { SD) }\end{array}$ & $5.97 \pm 4.76$ & $7.23 \pm 4.97$ & $6.6 \pm 4.87$ & $>0.05^{*}$ \\
\hline
\end{tabular}

\section{Change of Clinical Parameters after Nonsurgical Periodontal Treatment}

The clinical parameters (PII, GI, PPD, CAL) of both groups at baseline, third month, and sixth month are shown in Table 2. At baseline, there was no statistically considerable difference in periodontal indices between the two groups $(p>0.05)$. After nonsurgical treatment, the clinical parameters of Group I (OHI) and Group II $(\mathrm{SRP}+\mathrm{OHI})$ showed different changes.

At third month follow-up, the results showed that there was a reduction in $\mathrm{GI}(p<0.01)$ and an increase in CAL $(p<0.01)$ in Group I. Meanwhile, all the PII, GI, and CAL indexes of Group II exhibited a significant reduction $(p<0.01)$. The difference between the two groups in all indexes was statistically significant $(p<0.05)$.

After 6 months, all recorded indexes of Group II showed lower values as compared to those at baseline $(p<0.01)$. However, $\mathrm{OHI}$ (Group I) presented no effect on changing PlI, Gl, and PPD ( $p>0.05)$. Noticeably, the CAL values continuously increased from $1.97 \pm 0.4$ $\mathrm{mm}$ at baseline to $2.06 \pm 0.33 \mathrm{~mm}$ at third month, and $2.2 \pm 0.36$ at sixth month $(p<0.01)$. The difference between the two groups in all indexes was statistically significant $(p<0.05)$.

\section{Change of Hba1c Level after Nonsurgical Periodontal Treatment}

Regarding glycemic control, Group I showed an insignificantly different $\mathrm{HbA} 1 \mathrm{c}$ level $(7.06 \pm 0.74 \%)$ at the third month, as compared to that at baseline $(7.06 \pm 0.72 \%)$ (Fig. 1). At the 6-month visit, there was an increase in $\mathrm{HbA1c}$ level $(7.37 \pm 0.75 \%, p<0.01)$. Meanwhile, Group II, in which patients received SRP $+\mathrm{OHI}$, showed a reduction in $\mathrm{HbA1c}$ values from $7.34 \pm 0.78 \%$ at baseline to $7.01 \pm 0.64 \%$ at third month and $6.92 \pm 0.63 \%$ at 6 month $(p<0.01)$.

At baseline and third month recall, there was no significant difference in $\mathrm{HbA} 1 \mathrm{c}$ level between the two groups $(p>0.05)$. The difference in $\mathrm{HbA1c}$ level between the two study groups after 6 months was statistically significant $(p<0.05)$.

\section{Discussion}

In the management of $\mathrm{CP}$, nonsurgical periodontal therapy is administered to improve clinical parameters as well as control the bacterial count responsible for initiating the disease and the resultant inflammatory response. ${ }^{8}$ In the current study, 64 patients with T2DM and CP were distributed to two groups, each patient received one of two nonsurgical treatment modalities: Group I was provided $\mathrm{OHI}$, Group II was treated by SRP combining $\mathrm{OHI}$. The efficacy of the two modalities was presented through the change of clinical periodontal parameters and glycosylated hemoglobin values at 3 months and 6 months follow-up. 
Effects of Two Nonsurgical Periodontal Treatment Modalities in Diabetic Patients

Table 2: Comparison of clinical parameters at baseline, third month and sixth month between two groups

\begin{tabular}{|c|c|c|c|c|c|c|c|c|}
\hline Clinical parameters & Group & Baseline & Three months & $\Delta_{1}$ & $p_{1}$ & 6 months & $\Delta_{2}$ & $p_{2}^{*}$ \\
\hline & Group I & $1.64 \pm 0.25$ & $1.6 \pm 0.23$ & -0.04 & $>0.05$ & $1.68 \pm 0.29$ & 0.04 & $>0.05$ \\
\hline \multirow[t]{3}{*}{ PII } & Group II & $1.7 \pm 0.24$ & $1.2 \pm 0.25$ & -0.5 & $<0.01$ & $1.08 \pm 0.24$ & -0.62 & $<0.01$ \\
\hline & $p^{* *}$ & $>0.05$ & $<0.01$ & & & $<0.01$ & & \\
\hline & Group I & $1.6 \pm 0.27$ & $1.48 \pm 0.21$ & -0.13 & $<0.01$ & $1.52 \pm 0.25$ & -0.09 & $>0.05$ \\
\hline \multirow[t]{3}{*}{ GI } & Group II & $1.55 \pm 0.22$ & $1.1 \pm 0.22$ & -0.44 & $<0.01$ & $1.02 \pm 0.22$ & -0.53 & $<0.01$ \\
\hline & $p^{* *}$ & $>0.05$ & $<0.01$ & & & $<0.01$ & & \\
\hline & Group I & $1.2 \pm 0.15$ & $1.2 \pm 0.12$ & -0.004 & $>0.05$ & $1.21 \pm 0.15$ & 0.01 & $>0.05$ \\
\hline \multirow[t]{3}{*}{ PPD $(\mathrm{mm})$} & Group II & $1.21 \pm 0.27$ & $1.13 \pm 0.13$ & -0.087 & $>0.05$ & $1.06 \pm 0.17$ & -0.15 & $<0.01$ \\
\hline & $p^{* *}$ & $>0.05$ & $<0.05$ & & & $<0.01$ & & \\
\hline & Group I & $1.97 \pm 0.4$ & $2.06 \pm 0.33$ & 0.09 & $<0.01$ & $2.2 \pm 0.36$ & 0.24 & $<0.01$ \\
\hline \multirow[t]{2}{*}{$\mathrm{CAL}(\mathrm{mm})$} & Group II & $1.96 \pm 0.41$ & $1.81 \pm 0.39$ & -0.15 & $<0.01$ & $1.78 \pm 0.37$ & -0.18 & $<0.01$ \\
\hline & $p^{* *}$ & $>0.05$ & $<0.01$ & & & $<0.01$ & & \\
\hline
\end{tabular}

${ }^{*} p$ value obtained with the Wilcoxon signed-rank test for probing pocket depth and the paired samples $t$-test for other variables for intragroup changes from baseline

${ }^{* *} p$ value obtained with the Mann-Whitney U-test for probing pocket depth and the independent samples $t$-test for other variables for the intergroup comparison at the same time point

PII, plaque index; GI, gingival index; PPD, probing pocket depth; CAL, clinical attachment loss; Group I, oral hygiene instruction; Group II, scaling; root planing; and oral hygiene instruction

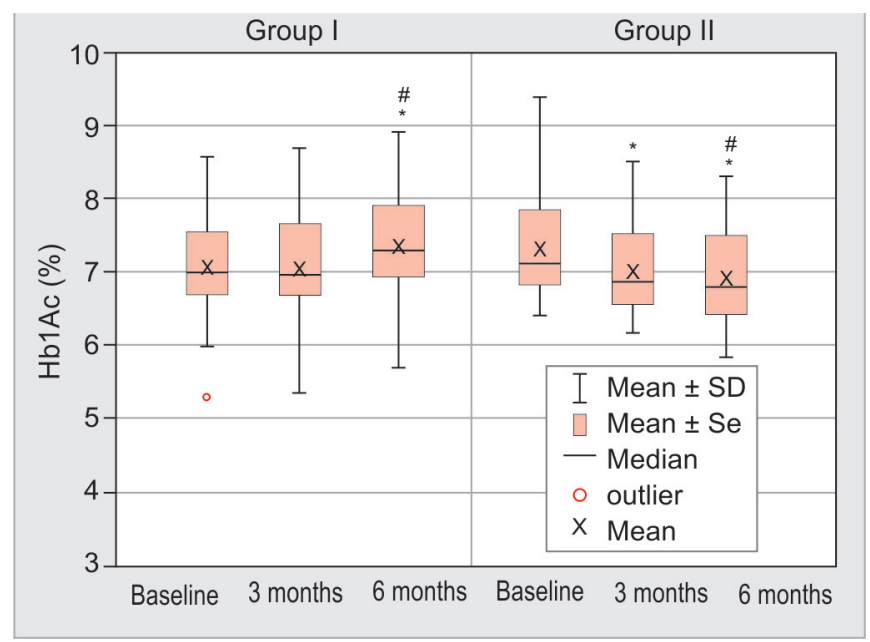

Fig. 1: Box-plot diagram exhibiting the HbA1c (glycated hemoglobin) levels (\%) at baseline, 3-month, and 6-month visits in each group. *Statistically significant difference in $\mathrm{HbA} 1 \mathrm{c}$ reduction levels compared to baseline of each group (Student's $t$-test; $p<0.05$ )

"Statistically significant difference in $\mathrm{HbA} 1 \mathrm{c}$ reduction levels between groups (Student's $t$-test; $p<0.05$ )

The obtained results showed that $\mathrm{OHI}$ did not achieve a significant effect on decreasing plaque formation in patients $(p>0.05)$. Brushing teeth with Bass technique and interdental cleaning using floss were instructed as daily self-performed. Most of the participants in this study are the elders ( $>65$ years old). It seems that brushing teeth with the Bass technique and interdental cleaning using floss might be difficult for Vietnamese elders to maintain good oral hygiene. A systematic review has recommended that $\mathrm{OHI}$ in addition to a professional session of prophylaxis, wellmotivated and proper instruction, powered toothbrushes might enhance plaque removal and reduce gingival inflammation. ${ }^{18}$ Following the unchanged PII, the GI of Group I exhibited a slight improvement in the third month $(p<0.01)$ and insignificant change in the sixth month. Noticeably, Group I patients showed an insignificant change of the PPD values, but an increase in the $\mathrm{CAL}$ values. After receiving $\mathrm{OHI}$ therapy, gingival inflammation of diabetic patients seems to be controlled, but the destruction of deep periodontium still progressed, The $\mathrm{OHI}$ therapy might only have effects on the removal of supragingival plaque. Meanwhile, the remaining subgingival plaque was the main reason leading to further attachment loss. In a study of Asad on CP patients including controlled MD, the $\mathrm{OHI}$ group showed a significant improvement in PPD and CAL only for shallow and moderately deep pockets at 3 months after treatment. ${ }^{19}$ Improvements in supragingival plaque control brought about by $\mathrm{OHI}$ might result in partial shrinkage of the gingiva and reduced probing depths at periodontally involved shallow sites but not deeper sites. ${ }^{20}$

Regards to $\mathrm{OHI}$ effects on DM patients, earlier studies have reported different results. In Artese et al.'s study, the supragingival therapy on DM patients showed a modest effect on periodontal clinical parameters, and a limited effect on systemic inflammatory markers, compared with the intensive periodontal therapy. Intensive periodontal therapy was suggested to be prioritized to manage the inflammatory burden on T2DM patients. ${ }^{21}$ Meanwhile, a control clinical trial in India by Kanduluru et al. showed that Gl, bleeding on probing (BOP), PPD, and CAL scores were significantly reduced at third-month follow-up in DM patients receiving $\mathrm{OHI} .{ }^{22}$ In a study of El-Makaky, if the patients with periodontitis and uncontrolled diabetes were not received any periodontal therapies (control group), the mean values of visible dental plaque, PPD, BOP, and CAL were significantly increased from baseline to end of the study. ${ }^{23}$ Summarized from previous studies, $\mathrm{OHI}$ might have a mild effect on idling or slowing down the periodontal inflammation in DM patients. In this study, $\mathrm{OHI}$ therapy could maintain the gingival status of DM patients in 6 months.

As compared to the $\mathrm{OHI}$ modality alone, SRP combining $\mathrm{OHI}$ showed better effects on improving all periodontal parameters at both third and sixth month follow-up visits $(p<0.05)$. Similar results were also reported in previous studies in diabetic patients: a significant reduction in microbial count and improvement of clinical variables in the SRP + OHI group, as compared to the $\mathrm{OHI}$ 
group. ${ }^{19,22}$ Thus, the $\mathrm{OHI}$ alone is not effective enough to manage the plaque formation and attachment loss, additional SRP played an important role in improving all periodontal parameters for DM patients.

In this study, to assess the effect of nonsurgical therapies on glycemic control, the $\mathrm{HbA1c}$ data were also collected at third- and sixth-month visits. The $\mathrm{OHI}$ showed no change of $\mathrm{HbA1c}$ level at the third month and an increase of this parameter at the sixth month. Meanwhile, there was a significant reduction of $\mathrm{HbA1c}$ after 3 months and 6 months in patients receiving SRP $+\mathrm{OHI}$ therapy. The combination of SRP and $\mathrm{OHI}$ has improved glycemic control $(p<0.05)$. The effectiveness of periodontal treatment for improving glycemic control in individuals with T2DM remains controversial. A recent retrospective cohort by Kim et al. showed that nonsurgical periodontal treatment had a maintenance effect on baseline $\mathrm{HbA1c}$ levels, while the control cohort had a steadily increasing trend from baseline to 6 and 12 months. ${ }^{24}$ The DIAPERIO randomized controlled trial of Vergnes et al. proposed that although periodontal treatment showed no clinical effect on glycemic control, its safeness and effects on improving oral health-related quality of life in diabetic patients were considered. ${ }^{25}$ Despite the different results, periodontal therapy had no negative effect on glycemic control in patients with diabetes.

Recently, the global burden of the COVID-19 pandemic has had a severe effect on health systems, becoming the third leading cause of death behind cancer and cardiovascular diseases in 2020 in America. ${ }^{26} \mathrm{~A}$ case-control study by Marouf et al. has found that periodontitis was related to a higher risk of ICU admission, need for ventilation, and death of COVID-19 patients. ${ }^{27}$ The uncontrolled hyperglycemia probably raises the risk of developing periodontitis and activating overexpression of ACE2 in periodontal tissue of T2DM patients. Then, the increase of ACE2 might lead to SARS-CoV-2 infection and the development of a mild-to-severe form of COVID-19. ${ }^{27}$

In Vietnam, as of 31 March 2021, a year since COVID-19 was declared a national pandemic, Vietnam had reported 2603 confirmed cases in 48 of the country's 63 provinces, including 35 deceased cases. ${ }^{28}$ This study was conducted during the COVID-19 pandemic in Vietnam when the social distancing policy was applied in many cities to lower the virus transmission and prevent the pandemic spread. As such, many DM patients found it difficult to reach the periodic examination. Moreover, dental cleaning, nonurgent oral health care, was advised to be delayed by the $\mathrm{WHO}$ and replaced by other remote dental care consultants. The obtained results might suggest that the $\mathrm{OHI}$ modality with a proper oral health care program can help DM patients with periodontitis control the gingival condition during pandemic outbreaks. Besides that, a professional dental debridement such as SRP is necessary for them to improve periodontal and glycemic status. To prevent the spread of the pandemic, strict screening for patients and modifications for dental operatories should be implemented..$^{15}$ Although periodontal therapy can be considered a contagious risk for diabetic patients, maintaining oral health care for DM patients with CP during the pandemic is reasonable. Moreover, an appropriate periodontal maintenance program, specific to individual circumstances, has been recommended to the patient for long-term control of the disease. ${ }^{29}$ Thus, irrespective of the prevalence of current COVID-19 pandemic, periodic examinations with nonsurgical periodontal treatments are necessary for DM patients with CP.

\section{Conclusion}

The findings from this study confirm that nonsurgical periodontal treatment has positive effects on controlling periodontitis and glycemic levels in diabetic patients. In addition to the $\mathrm{OHI}$, the SRP modality must be taken into consideration for improving periodontal disease in patients with diabetes.

\section{Acknowledgment}

This study was approved by the Institutional Ethics Committee of Hue University of Medicine and Pharmacy, Hue University (Number H2020/035).

\section{Authors' Contribution}

All authors participated in the design of the study, data collection, and interpretation of the data, and drafted the manuscript. All authors read and approved the final manuscript.

\section{References}

1. Internation Diabetes Federation. IDF diabetes atlas ninth. Dunia: IDF. 2019.

2. Wu C-Z, Yuan Y-H, Liu H-H, W, et al. Epidemiologic Relationship Between Periodontitis and type 2 Diabetes Mellitus. BMC Oral Health 2020;20(1):1-15. DOI: 10.1186/s12903-020-01180-w.

3. Oana AV, Caroline K, Dan O, et al. Diabetes Mellitus, and Periodontal Disease-a Two-Way Road: Current Concepts and Future Considerations (Literature Review). ESJ 2013;9(9):62-79. DOI: 10.19044/esj.2013.v9n9p\%p.

4. Singh $M$, Bain $B K$, Jhingran $R$, et al. Prevalence of Periodontal Disease in type 2 Diabetes Mellitus Patients: A Cross Sectional Study. Contemp Clin Dent 2019;10(2):62-79. DOI:10.4103/ccd.ccd_652_18.

5. Genco RJ, Borgnakke WS. Diabetes as a Potential Risk for Periodontitis: Association Studies. 2020;83(1):40-45. DOI: 10.1111/prd.12270.

6. Saini R, Saini S, Sugandha R. Periodontal Disease: The Sixth Complication of Diabetes. J Fam Commun Med 2011;18(1):31. DOI: 10.4103/1319-1683.78636.

7. Chang P C, P LL. Interrelationships of Periodontitis and Diabetes: A Review of the Current Literature. Assoc Dental Sci Republ Chin 2012;7(3):272-282. DOI: 10.1016/j.jds.2012.02.002.

8. Plessas A. Nonsurgical Periodontal Treatment: Review of the Evidence. Oral Health Dental Manage 2014;13(1):71-80. PMID: 24603920.

9. Sandhu HS, Jaffer A. Diabetes and Periodontitis: Multidisciplinary Management. Integrat Molecul Med 2016;3(2):612-615. DOI: 10.15761/ IMM.1000212.

10. Chapple ILC, Genco R, workshop wgotjEA. Diabetes and periodontal diseases: consensus report of the Joint EFP/AAP Workshop on Periodontitis and Systemic Diseases. 2013;40(S14):S106-S12. DOI: 10.1902/jop.2013.1340011.

11. Baeza M, Morales A, Cisterna C, et al. Effect of Periodontal Treatment in Patients with Periodontitis and Diabetes: Systematic Review and Meta-analysis. J Appl Oral Sci 2020;28:e20190248-e. DOI: 10.1590/1678-7757-2019-0248.

12. Casillas Santana MA, Arreguín Cano JA, Dib Kanán A, et al. Should We Be Concerned about the Association of Diabetes Mellitus and Periodontal Disease in the Risk of Infection by SARS-CoV-2? Syst Rev Hypoth Medicina (Kaunas, Lithuania). 2021;57(5):493. DOI: 10.3390/ medicina57050493.

13. Morales A, Corral-Nuñez C, Galaz C, et al. Impact of COVID-19 Pandemic on Quality of Life of Type 2 Diabetes Patients With Periodontitis. Front Oral Health 2021;2(29):682219. DOI: 10.3389/ froh.2021.682219.

14. World Health Organization. Considerations for the Provision of Essential Oral Health Services in the Context of COVID-19: 
Interim Guidance, 3 August 2020. Geneva: World Health Organization; 2020.

15. Jafer MA, Hazazi MA, Mashi MH, et al. COVID-19 and Periodontitis: A Reality to Live with. J Contemp Dent Pract 2020;21(12):1398-1403. DOI: 10.5005/jp-journals-10024-2961.

16. American Diabetes Association. Classification and Diagnosis of Diabetes: Standards of Medical Care in Diabetes-2020. Diabetes Care 2020;43(S1):S14-S31. DOI: 10.2337/dc20-S002.

17. American Academy of Periodontology. Task Force report on the Update to the 1999 Classification of Periodontal Diseases and Conditions. J Periodontol 2015;86(7): 835-838. DOI: 10.1902/jop.2015.157001.

18. van der Weijden GA, Hioe KP. A Systematic Review of the Effectiveness of Self-performed Mechanical Plaque Removal in Adults with Gingivitis Using a Manual Toothbrush. J Clin Periodontol 2005; 32(S6):214-228. DOI: 10.1111/j.1600-051X.2005.00795.x.

19. Asad M, Abdul Aziz AW, Raman RPC, et al. Comparison of Nonsurgical Periodontal Therapy with Oral Hygiene Instruction Alone for Chronic Periodontitis.J Oral Sci 2017;59(1):111-120. DOI: 10.2334/josnusd.16-0298.

20. Umeda M, Takeuchi $Y$, Noguchi K, et al. Effects of Nonsurgical Periodontal Therapy on the Microbiota. Periodontology 2004;36: 98-120. DOI: 10.1111/j.1600-0757.2004.03675.x.

21. Artese HP, Longo PL, Gomes GH, et al. Supragingival Biofilm Control and Systemic Inflammation in Patients with type 2 Diabetes Mellitus. Brazil Oral Res 2015;29(1):1-7. DOI: 10.1590/1807-3107BOR-2015. vol29.0071.
22. Kanduluru A, Naganandini S. Effect of Nonsurgical Periodontal Treatment on Clinical Response and Glycemic Control in type 2 Diabetic Patients with Periodontitis: Controlled Clinical Trial. J Indian Assoc Public Health Dent 2014;12(4):261-267. DOI: 10.4103/ 2319-5932.147643.

23. El-Makaky Y, Shalaby HK. The Effects of Non-Surgical Periodontal Therapy on Glycemic Control in Diabetic Patients: A Randomized Controlled Trial. Oral Dis 2020;26(4):822-829.

24. Kim S-H, Lee J, Kim W-K, et al. HbA1c Changes in Patients with Diabetes Following Periodontal Therapy. J Periodontal Implant Sci 2021;51(2):114-123.

25. Vergnes JN, Canceill T, Vinel A, et al. The Effects of Periodontal Treatment on Diabetic Patients: The DIAPERIO Randomized Controlled Trial. J Clin Periodontol 2018;45(10):1150-1163.

26. Woolf SH, Chapman DA, Lee JH. COVID-19 as the Leading Cause of Death in the United States. Journal of the American Medical Association 2021;325(2):123-124.

27. Marouf N, Cai W, Said KN, et al. Association between Periodontitis and Severity of COVID-19 Infection: A Case-Control Study. J Clin Periodontol 2021;48(4):483-491.

28. Nguyen TDT, Kawai K, Nakakubo T. Estimation of COVID-19 Waste Generation and Composition in Vietnam for Pandemic Management. Waste Manage Res 2021;39(11):1356-1364.

29. American Academy of Periodontology. Guidelines of Periodontal Therapy. J Periodontol 2001;72:1624-1628. 